\title{
Recomposición familiar y transformaciones agrarias. Lectura de dos casos africanos y uno mexicano
}

\author{
André Quesnel* \\ Patrice Vimard**
}

Los autores de este trabajo discuten el papel que desempeña el régimen demográfico, y par. ticularmente sus cambios, en las transformaciones agrarias. La hipótesis que guia la investigación cuyos resultados se exponen aqui, es que la estructura demográfica constituye un factor explicativo pära dar cuenta del funcionamiento y reproducción de diversas instancias sociales. Interesa en particular a los autores repensariel debate en torno al papel de la innovación en la relación entre población y transformaciones productivas.

Aun cuando se sostiene que las transformaciones agrarias, que tienen lugarien las regiones aqui analizadas, son un resultado de cambios demográficos, se destaca también que éstas resultan influidas por el contexto social mayor del que forman parte, de tal manera que las relaciones con la economia de mercado, con la sociedad urbana y con el trabajo asalariado constituyen factores que deben ser tomados en cuenta para teneri una explicación más comprensiva de las influencias reciprocas entre lo demográfico, lo tecnológico y lo socioeconómico.

\section{Introducción ${ }^{1}$}

La cuestión de la innovación ha permanecido en el centro del debate sobre las evoluciones demográfica y agraria desde que éste fue reabierto por Ester Boserup en 1965. A partir de su tesis, según la cual la presión demográfica genera innovaciones tecnológicas y permite el desarrollo de la producción agrícola (Boserup, 1965, 1970), los economistas, historiadores y agrónomos han emprendido numerosos estudios en contextos -y con escalas de tiempo y espacio- extremadamente distintos. ${ }^{2}$ En los ejemplos y con traejemplos presentados, el

* Demógrafo, director de investigación en orstom, miembro de la unidad de investigación "Peuplement et dynamique sociale des espaces ruraux" ("Poblamiento y dinámica social de los espacios rurales"), del departamento Milieux et activité agricole (Medios y actividad agricola).

** Demógrafo, director de investigación en orstom, miembro del equipo de investigación "Transition de la fecondité et santé de la reproduction" ("Transición de la fecundidad y salud de la reproducción"), UR36, departamento MAA, LPE, Cenre St. Charles, 13331 Marseille Cedex 3.

${ }^{1}$ Agradecemos a los lectores anónimos y a Marc Pilon (orstom-CEPED) los comentarios que amablemente hicieron a las primeras versiones de este texto. Nos declaramos los únicos responsables del análisis desarrollado aquí.

${ }^{2}$ La tesis de Boserup sigue sirviendo como marco de referencia a ciertos estudios. Muchos autores la han citado explícitamente, como en las obras de Francis Gendreau 
régimen demográfico de la población suele expresarse en términos de variables exógenas como tamaño, densidad y, en el mejor de los casos, ritmo de crecimiento, tratando de establecer su relación causal con las transformaciones agrarias, mientras que la aportación fundamental de Ester Boserup fue restituir la endogeneidad a la dinámica demográfica en la evolución de los sistemas agrarios.

Partiendo de este punto de vista y manteniendo que el régimen demográfico regula el funcionamiento y reproducción de todas las instancias sociales, parece necesario tomar en cuenta más particularmente, bajo el efecto de un cambio de régimen, las transformaciones que tienen lugar en instancias como la unidad de explotación y, de manera más general, la unidad familiar, que presiden la producción, si se quiere aprehender toda forma de innovación. Además se necesita superar el sentido económico estricto de innovación, según el cual ésta es el medio, técnico u organizacional, para incrementar la productividad de la firma, la unidad de producción o la explotación agrícola. Ya sea social o institucional, la innovación es más frecuentemente un ajuste inédito que busca mitigar una disfunción que afecta a una organización social en conjunto. Es necesario asimismo superar la noción de ciclo familiar de la unidad de explotación, tal y como se ha desarrollado a partir de Anton Chayanov (1966, 1990), quien consideraba la unidad de explotación campesina como el sitio de asignación de los recursos y, más particularmente, de la fuerza de trabajo con el fin de satisfacer las necesidades del grupo. En este marco, la innovación no puede emerger en la medida en que la estructura demográfica define el horizonte de las necesidades y en que la unidad doméstica, al redistribuir su fuerza de trabajo en su interior, no es cuestionada. Ahora bien, aunque parece que la familia campesina es el lugar donde se amortiguan las crisis económicas y demográficas, es necesario admitir igualmente que volver a cuestionar los arreglos familiares en cuanto a su tamaño, composición y ciclo conlleva a su vez una transformación de la producción, de los modelos familiares y de las conductas reproductivas.

También hay que reconocer ante todo que es la integración al mercado, según distintas formas e intensidades, así como el control

et al. (1991) y Antoinette Fauve-Chamoux (1987). Aquí hacemos referencia al modelo inicial de Boserup (1965), cuyo funcionamiento se establece para un medio cerrado; la misma Boserup ha reconocido los límites del modelo en cuanto las poblaciones reciben oportunidades externas a su medio (1991). 
ejercido por el Estado sobre el mercado de bienes y de trabajo, lo que engendrará la transformación de la organización de la producción, de las instancias que la presiden y, más globalmente, de las relaciones sociales que allí se establecen entre los individuos. Haciendo esto se subraya que los cambios de régimen demográfico de las sociedades rurales están vinculados a las relaciones que establecen con el mercado, el mundo urbano y el asalariado. Así, parece que sólo entonces conviene considerar los reajustes que provoca todo cambio de régimen demográfico en el dominio de la producción, aunque también en otros dominios, como el de las relaciones sociales entre generaciones y entre los individuos dentro de la familia.

Siguiendo esta línea, queremos mostrar aquí el papel de la transformación de los ciclos y arreglos familiares en la evolución agraria, apoyándonos en el ejemplo de sociedades rurales de África occidental, en Costa de Marfil y Togo, cuyas transformaciones agrarias están vinculadas en primer lugar con el desarrollo de los cultivos comerciales controlados por el Estado, y que experimentan desde hace veinte años un crecimiento demográfico sin precedentes en su historia.

El análisis de esta situación africana hará referencia esencialmente a dos regiones situadas en zonas selváticas propicias para cultivos comerciales como café y cacao, cuya producción se desarrolló durante la colonización francesa y más incluso después de la independencia, en la segunda mitad del siglo. Se hará referencia por una parte a la región de la meseta de Daye al suroeste de Togo, donde se puede distinguir entre las poblaciones autóctonas, de etnia -ewey ahlon-, propietarias de sus plantaciones, y las poblaciones inmigrantes del norte del país, especialmente de etnia kabye, empleados como braceros o aparceros. Por otra parte se hablará de la región de Sassandra, en el suroeste de Costa de Marfil, poblada en su mayoría por inmigrantes venidos del conjunto de África occidental a trabajar en uno de los últimos frentes pioneros del subcontinente. ${ }^{3}$

De manera simultánea se examinará la sociedad rural del estado de Yucatán, al sureste de México, cuya producción de henequén (sisal) está controlada por el Estado y que se encuentra en un punto

${ }^{3}$ Los datos utilizados para estos análisis provienen de observaciones realizadas asociando encuestas demográficas en los hogares con entrevistas a mujeres en edad fértil y a hombres migrantes o encargados de la explotación agrícola. Estas observaciones corresponden para la meseta de Daye al periodo de 1970-1979 y para la región de Sassandra a los años de 1988-1993. 
avanzado de su transición demográfica. Los distintos procesos históricos de la península de Yucatán después del siglo xvir y el desarrollo de la producción de henequén a partir del siglo XIx han concurrido en una concentración de la población en la zona henequenera de Yucatán. Ésta reúne a la mayor parte de la población del estado (más de $75 \%$ en 1980), de la cual cerca de la mitad vive en Mérida, la capital regional. Por esto, y con el control directo de su producción agrícola, la población rural de la zona henequenera ocupa un lugar particular en el conjunto cultural maya de la cuenca mesoamericana: por una parte, aunque la lengua maya se sigue utilizando de manera corriente, la proporción monolingúe de la población se ha reducido mucho; por otra parte, no se encuentran las formas evidentes de organización social y productiva de la sociedad maya contemporánea de otras zonas rurales, tal y como se han descrito en los trabajos de los antropólogos. También nos parece vano, si no es que falaz, buscar una "influencia cultural" cualquiera sobre los modos actuales de organización familiar, aunque sin negar la noción de marcador de identidad, sino todo lo contrario, pues la población rural de la zona henequenera nos interesa aquí por las restricciones productivas y demográficas que han inducido todo un trabajo de recomposición familiar en esta última parte del siglo. ${ }^{4}$

En los tres casos considerados mostraremos que la innovación en el dominio agrícola no puede proceder directamente de la presión demográfica, en la medida en que las nuevas prácticas familiares responden tanto a las transformaciones agrarias ligadas a la integración creciente al mercado, como a los efectos del rápido crecimiento demográfico que acompaña a dicha integración. Depende de los reacomodos que se realizan, de acuerdo con un calendario ${ }^{5}$ muy corto, en la organización de la unidad familiar y de la unidad de explotación. Por esto la innovación será antes que nada de orden social y en la mayoría de los casos volverá caducos los constreñimientos que hubieran podido conducir a progresos de orden técnico.

\footnotetext{
${ }^{4}$ Los resultados utilizados se extrajeron de un estudio realizado en 1980-1981 en el marco del proyecto "Cambios demográficos en diferentes zonas agrarias de México", bajo la dirección de Susana Lerner (Colmex) y André Quesnel (Orstom).

${ }^{5}$ Por otra parte, y de manera general, es la consideración del calendario o de la temporalidad específicos y muy diferenciados de los procesos demográficos, sociales, técnicos y ecológicos lo que permite en parte identificar el sentido y el carácter de los lazos de causalidad entre los distintos procesos.
} 
El papel de la integración al mercado

Del linaje al grupo doméstico: el repliegue sobre una unidad restringida de control de la:producción en África subsahariana

Las transformaciones más rápidas y más importantes en la organización familiar ${ }^{6}$ ocurrieron con la constitución de los "sistemas de economía de plantación" desarrollados en los macizos selváticos del oeste africano, en Costa de Marfil y Togo (Quesnel y Vimard, 1988; FausseyDomalain y Vimard, 1991), donde se impuso la asignación de un máximo de tierras y sobre todo de mano de obra a la producción de cultivos comerciales.

El papel fundamental de la apropiación de la mano de obra en el funcionamiento de la economía de plantación es concomitante con un desplazamiento del control de la reproducción y de la utilización de la fuerza de trabajo, del linaje hacia un grupo doméstico restringido a un solo componente nuclear complementado a la larga con algunos parientes jóvenes (colaterales, nietos). Dentro de la unidad familiar la matriz de la fuerza de trabajo concierne a los dependientes inmediatos de los jefes de grupos domésticos autóctonos, que han tenido la iniciativa individual de crear plantaciones; fuera de ella se extiende a la mano de obra alógena proveniente de las regiones de las sabanas, donde no se han podido introducir cultivos perennes por razones agronómicas y climáticas.

En cuanto a las poblaciones inmigrantes, utilizan de golpe una organización de la producción basada igualmente en una unidad doméstica restringida. En efecto, el jefe de una familia alógena, separada de su linaje de origen, se encuentra en la situación de ser el único

\footnotetext{
${ }^{6}$ En este texto se considera como grupo doméstico el conjunto de individuos que comparten la misma habitación y consumen en común. Este grupo doméstico es asimismo una unidad de producción, ya sea totalmente, cuando todos sus miembros ejercen su actividad en una misma explotación, o parcialmente, cuando algunos de los miembros que lo componen ejercen sus actividades al exterior. Esta definición corresponde igualmente a la de hogar. Se dice que un hogar es nuclear cuando sus miembros pertenecen todos a la familia biológica delijefe de familia, o sea, su(s) mujere(s) y sus hijos. En cambio, también se pueden identificar en un hogar uno o varios componentes nucleares, constituidos cada uno por un hombre, su(s) mujere(s) y sus hijos. La unidad de producción o de explotación representa al conjunto de los individuos que se organizan para producir en común de manera permanente; la unidad de consumo está constituida por las personas cuyo autoconsumo alimenticio se extrae de una misma reserva de producto. En cuanto a la unidad familiar, o la familia, está constituida por el conjunto de los miembros unidos por lazos sanguíneos, maritales o de adopción.
} 
responsable de la organización de la producción y se beneficia de manera exclusiva de la fuerza de trabajo que representan su mujer y sus hijos (Quesnel y Vimard, 1987).

Esta innovación no es exclusiva de las agrupaciones insertadas directamente en la producción de los cultivos comerciales, sino que concierne también a las poblaciones ligadas a este sistema de economía mercantil por la reproducción de una fuerza de trabajo que será utilizada en las plantaciones. Se puede presentar aquí el ejemplo de los mossi de Burkina Faso, entre los cuales se nota una desagregación de los linajes y segmentos de linaje y un fraccionamiento de las unidades familiares de explotación agrícola de antaño, que correspondían a grupos particularmente extendidos de personas emparentadas. También para estas comunidades el grupo doméstico se ha vuelto con frecuencia la unidad de producción y de consumo y la separación de unidades independientes "vence hoy en día al ajuste de las unidades jerarquizadas" (Marchal, 1987). Este fenómeno es menos neto entre los moba del norte de Togo, donde la familia extendida era más una instancia de control de la producción que de organización efectiva de la explotación agrícola, a pesar de lo cual se nota también una dilución del control en beneficio de grupos domésticos más restringidos (Pilon, 1989).

Expansión y fragmentación del espacio familiar por la multiplicación de los componentes nucleares en las unidades domésticas en Yucatán

Para la mayor parte de las sociedades rurales de América Latina se admite que en el periodo contemporáneo el repliegue en una unidad doméstica restringida ha acompañado la integración al mercado, y tanto más fácilmente cuanto que desde la conquista numerosos factores concurrieron en ello: ${ }^{7}$ la imposición del matrimonio cristiano a partir del periodo colonial, el endeudamiento de los campesinos y su correlato, el peonaje en el régimen de haciendas hasta finales del siglo xix y, en México, la parcelación de las tierras con la reforma agraria después de la revolución (Bernard y Gruzinski, 1986). No obstante, en los años 1960-1970 la importante proporción de unidades domésticas no nucleares en las numerosas regiones rurales de Méxi-

${ }^{7}$ A la inversa, las familias rurales que migran a la ciudad se ven a menudo obligadas a reagruparse en una misma unidad residencial. 
co, como Yucatán, regularmente se ha interpretado, por una parte, como la persistencia de un modelo familiar vertical, patrilineal y virilocal y, por la otra, como el desarrollo de una organización correspondiente a una "estrategia de supervivencia" y de mantenimiento de la explotación agrícola (Pantelides, 1984). De hecho, la formación de unidades domésticas extendidas -por la conservación del componente nuclear de uno o varios de los hijos del responsable del grupo doméstico- remite más a la dificultad de muchos hombres casados para formar unidades autónomas por falta de acceso a la tierra, a un traba:jo asalariado estable, a una vivienda individual, etc., que a una necesidad y una voluntad reales de organizarse como unidad de producción extendida. En Yucatán la complejidad creciente de los arreglos familiares no obedece a una necesidad de mano de obra familiar que se movilizaría de manera continua. Por el contrario, el responsable del grupo doméstico extendido se satisface con la rotación de la mano de obra dentro de su propio componente nuclear con el fin de asegurar la producción de la cantidad de henequén exigida por el Banco Rural. Esta cantidad disminuye en la medida en que el Banco Rural no puede aumentar los subsidios que otorga a los productores de este cultivo, a causa de la baja en el mercado internacional de las ventas tanto del henequén como de los productos manufacturados (Lerner y Quesnel, 1989).

En consecuencia, el acceso a la categoría de explotador de henequén, e incluso de explotador agrícola, es cada vez más difícil. Se percibe por esto una coexistencia prolongada de componentes nucleares emparentados (del padre, de los hermanos o de los hijos del:jefe de la unidad doméstica) en torno a una misma explotación agrícola. A medida que estos componentes se hacen más numerosos, debido a la disminución de la mortalidad, como se verá más adelante, sus responsables se vuelven hacia actividades independientes de aquellas ejercidas por los responsables de los otros núcleos familiares y por el jefe del grupo doméstico. Los más jóvenes son asalariados fuera del sector agrícola, los más ancianos se consagran exclusivamente a su explotación del henequén y los demás, la mayoría, se dedican a actividades diversas dentro y fuera del sector agrícola. Así, ya sea que se mantengan o se fragmenten las unidades de explotación, éstas están dirigidas por un hogar nuclear en torno del cual gravitan numerosos parientes, sobre todo los hijos casados que se dedican a diversas actividades de la producción agrícola (Lerner y Quesnel, 1985, 1989). De esta manera se percibe una fragmentación más importante del espacio fa- 
miliar, desde el punto de vista de la organización de sus actividades, que acompaña a su expansión. Esta fragmentación se confirma cuando los núcleos familiares, cada vez en mayor número, dejan su unidad doméstica de origen. No obstante, en Yucatán esto ocurre dentro del mismo espacio geográfico, ${ }^{8}$ a pesar del deterioro de la producción y comercialización del henequén, de las sucesivas reducciones del número de acreditados en el Banco Rural y del débil desarrollo de nuevas oportunidades de empleo en los sectores de actividad agrícola y no agrícola. Esto significa que la unidad familiar de origen constituye una base o un lugar de refugio o repliegue para losjóvenes yucatecos que se desplazan esencialmente dentro de su zona cultural.

La transformación de las relaciones sociales y de producción dentro de la familia

Una nueva forma de matrimonio: de la alianza entre grupos sociales a la unión interindividual

En África la autonomía adquirida por las unidades domésticas restringidas, en el proceso del trabajo agrícola, contribuye a una pérdida de control de los linajes sobre las alianzas matrimoniales. En efecto, los esposos y padres apartan a las mujeres y los niños de la organización por linaje de la producción, para destinarlos a los cultivos comerciales en beneficio único de la unidad doméstica a la que pertenecen. También la relación social entre el jefe del grupo doméstico y su linaje, y entre este linaje y otro, simbolizado por la mujer, pierde toda referencia a la producción y a la reproducción de la fuerza de trabajo. El matrimonio deja entonces de ser un intercambio o una alianza entre grupos sociales, para convertirse en una unión entre individuos. ${ }^{9}$ Esta pérdida de control de los linajes sobre las alianzas matrimoniales determina su decadencia, haciendo desaparecer la compensación matrimonial y la reciprocidad en la atribución de la descendencia de

$891 \%$ de los descendientes, $90 \%$ de los ascendientes y $80 \%$ de los hermanos del :jefe del grupo doméstico viven en la misma localidad que éste.

${ }^{9}$ El aumento de las uniones libres en detrimento de los matrimonios es rápida. Por ejemplo, en la meseta de Daye (suroeste de Togo), una zona de economía a base de plantaciones cafetaleras, las uniones por consenso mutuo han pasado de $20 \%$ (1930-1939) a 69\% (1975-1976), mientras que los matrimonios tradicionales han disminuido en el mismo periodo de $77 \%$ a $31 \%$ de las uniones (Quesnel y Vimard, 1988). 
las uniones, que representaban los mayores fundamentos del poder y del crecimiento de los linajes en el África negra (Augé, 1971). Este debilitamiento de los linajes favorece la autonomía de los individuos y engendra nuevas formas de relaciones sociales y de producción tanto entre hombres y mujeres como entre padres e hijos dentro de los grupos domésticos. Por otra parte, las prácticas individuales -como por ejemplo el rechazo de las muchachas al matrimonio obligatorio-, vinculadas con la difusión de nuevos valores, la promulgación de nuevas legislaturas y la intervención local de las misiones cristianas acentúan el debilitamiento de los controles ejercidos por los ancianos de los linajes.

Pero, a la inversa, cuando la expansión territorial y la movilización de una mano de obra extrafamiliar se vuelven más difíciles, el control de las uniones por parte de losijefes del grupo doméstico y el respeto de las prestaciones matrimoniales condicionan -para los hombresijóvenes- la creación de una plantación perenne y el mantenimiento de su explotación, asegurando la estabilidad con la producción de sus mujeres e hijos, única mano de obra movilizable. Por ejemplo, actualmente en el frente pionero del suroeste de Costa de Marfil, los hombresijóvenes se sujetan a este control familiar de su unión porque les sería difícil concebir la creación de una plantación sin el apoyo de sus padres, en cuanto al acceso a un terreno, y sin la ayuda de su mujer, en lo que se refiere a la limpieza de la plantación y a la producción alimenticia. ${ }^{10}$

Individuación de las relaciones entre hombre y mujer y el papel creciente de mujeres y niños en la producción agrícola

Al encontrarse en esencia circunscritas a un marco familiar restringido, las relaciones entre hombres y mujeres representan actualmente lazos individualizados entre, principalmente, el esposo y su(s) espo$\mathrm{sa}(\mathrm{s})$; las relaciones de poder, particularmente aquellas que expresan la dominación masculina, no se diluyen en el conjunto de linaje o el conjunto familiar extendido, sino que se encuentran personalizadas dentro de la unidad conyugal.

Las transformaciones en el proceso de producción repercuten de manera directa sobre la organización familiar. Cuando elijefe del grupo doméstico controla la mano de obra familiar durante la ejecución

${ }^{10}$ Comunicación personal de Éric Léonard. 
de los trabajos agrícolas, se ve, por una parte, investido del control de la reproducción demográfica efectuada al interior de su grupo y, por otra, obligado a cubrir los cargos que pesan sobre el grupo doméstico, en particular aquéllos ligados a la educación y salud de los hijos. Estas transferencias del control de la reproducción demográfica, de la retribución del trabajo agrícola y de la educación de los hijos, de los caciques de los linajes hacia losijefes de los grupos domésticos, activan la transformación de las relaciones entre los cónyuges.

La individuación de las relaciones de poder entre los sexos conlleva, las más de las veces, un reforzamiento de la autoridad masculina y, como corolario, una voluntad creciente de autonomía por parte de las esposas, como cuando los hombres no remuneran su trabajo ni cubren los gastos de educación de sus hijos. Esto se traduce particularmente en un aumento de la inestabilidad matrimonial, ${ }^{11}$ que se inscribe en el contexto general de debilitamiento del control familiar y de mayor libertad de los individuos. Así, el carácter y el sentido del matrimonio se transforman y se modifica el equilibrio de las estructuras matrimoniales. El ritmo de la constitución y disolución de las uniones se acelera y la duración de las uniones disminuye, con un efecto directo sobre la fecundidad y la estructura de las familias y de las unidades de explotación. ${ }^{12}$

La situación económica de las mujeres se ve igualmente modificada por la inserción en la economía de mercado. Si bien la propiedad personal de los productos del trabajo y la separación de los presupuestos y de los bienes eran la regla en las sociedades tradicionales (Paulme, 1952), casi no ofrecen beneficios a las mujeres en las economías agrícolas de subsistencia, donde pocos productos están comercializados. Pero todo esto cambia cuando se imponen las formas productivas y las actividades comerciales, en las que las mujeres asumen una parte preponderante en la transformación y comercialización de los productos alimenticios. Esto se aplica al medio rural y tanto más

${ }^{11} \mathrm{La}$ imposibilidad de satisfacer las necesidades de la familia se ha vuelto una de las causas más frecuentes de divorcio aducidas por las mujeres en estas sociedades. Esta inestabilidad afecta particularmente a las uniones consensuales, cuya naturaleza expresa la menor integración de los cónyuges a los marcos tradicionales de la nupcialidad (Quesnel y Vimard, 1988; Capron y Kohler, 1975).

12 En Daye se nota también, de manera concomitante en las etnias autóctonas, un aumento de las uniones por consentimiento mutuo, de la inestabilidad de los matrimonios y del número de mujeresıjefes de familia o que viven en casa de sus padres después de un divorcio, así como una disminución en el tamaño de las familias y de las unidades de explotación y una baja en la fecundidad (Quesnel y Vimard, 1988). 
en las ciudades, ya que la monetarización de la economía es más antigua y está más desarrollada. Esta independencia económica, procedente de la organización social tradicional, explica por quê las mujeres africanas han cobrado tal peso en las actividades mercantiles, donde controlan la mayor parte del comercio de mercado (Boserup, 1983).

Numerosos ejemplos subrayan la capacidad de una evolución económica favorable de las organizaciones familiares africanas originales en el marco de la economía de mercado. ${ }^{13}$ Sin embargo, esto no debe hacer olvidar que la creciente autonomía de las mujeres suele acompañarse de grandes dificultades para asegurar la supervivencia de su familia. Así, en un contexto de emigración masculina y de desarrollo de perímetros irrigados en Pays Toucouleur (valle medio de Senegal), Michéle Fiéloux (1985: 334) nota una regresión en relación con las mujeres de la sociedad tradicional: "más explotadas de lo queijamás lo habían estado, se han vuelto menos autónomas económicamente". Pero, paradójicamente, en el contexto de modernización agrícola que beneficia a los hombres, ellas son conducidas -perdiendo una parte de su autonomía, nos dice Michẻle Fiêloux-a tomar iniciativas personales en el dominio de la producción alimenticia, de la cual se encargan cada vez más: cambio de técnicas culturales, introducción de nuevas variedades, etc. He aquí la crisis, producto de una desestabilización en las relaciones entre hombres y mujeres, que suscita las innovaciones agrícolas.

La intensificación del trabajo de las mujeres se traduce las más de las veces en una extensión de las superficies cultivadas: ${ }^{14}$ en los cultivos comerciales ellas se sitúan cada vez más en posición de trabajador libre y agregan cultivos alimenticios personales a la remuneración de su trabajo en las plantaciones; en una economía de subsistencia, ellas deben cumplir con los trabajos en los campos colectivos para poder beneficiarse en los campos personales. Además, Jean-Yves Marchal (1987: 451) notó entre los mossi su voluntad de "asegurarse el máxi-

${ }^{13}$ Un ejemplo extremo lo ofrece el éxito económico de las comerciantes de hortalizas y productos pesqueros de Dakar, quienes emplean como asalariados a sus esposos, hortelanos o pescadores, estableciendo asi una forma asombrosa de contrato económico entre cónyuges, contraria a aquella consagrada por la tradición, que asegura la preponderancia masculina (Le Cour Grandmaison, 1979).

${ }^{14}$ Inversamente, la necesidad de aumentar las superficies cultivadas, ligada al desarrollo de los cultivos perennes y al crecimiento demográfico, conlleva una intensificación del trabajo de las mujeres. 
mo de independencia alimenticia" tomando prestadas parcelas de personas que no fueran sus cónyuges.

Es claro que las innovaciones de las mujeres se inscriben en el marco de las actividades compensatorias a las actividades de los hombres, dentro o fuera del sector agrícola, aspirando esencialmente a nuevas distribuciones de sus diferentes tiempos de trabajo. Así, las mujeres moba y gurma reducen el tiempo de barbecho de los campos próximos y ocupan las hondonadas: otras tantas iniciativas que amenazan la renovabilidad de los recursos, lo cual podrá conducir más tarde a nuevos cambios, como la introducción de nuevos cultivos o nuevas variedades. Al mismo tiempo, la participación de las mujeres en los grupos colectivos de ayuda mutua les permite un acceso a redes de información ajenas a su grupo familiar, que pueden ser fuente , de innovaciones sociales o agrícolas ulteriores (Pilon, 1991).

Las mujeres como responsables de la economía del grupo doméstico en Yucatán

En Yucatán la producción agrícola ya no es la actividad primordial alrededor de la cual se organiza la vida social y económica de la familia y del conjunto de la comunidad campesina. El matrimonio ya no sanciona la alianza de las familias en el tiempo y en torno a la producción, y el control de las familias sobre las uniones se vuelve mucho más laxo en la medida en que pierden su fundamento material. No obstante, el abandono de ciertas prestaciones matrimoniales, como el trabajo del esposo en la explotación de su suegro durante el primer año de matrimonio, $y$, en cambio, la residencia frecuente de laijoven paraja yucateca con la familia del hombre, colocan a la mujer cada vez más bajo su influencia ideológica al principio de la constitución de la descendencia, lo cual no dejará de influir en las relaciones de la pareja y de modificar la trayectoria reproductiva de la mujer. Pero al mismo tiempo, y en relación con lo que interesa aquí, al estar albergada por los padres de su esposo, la mujer se crea un espacio doméstico distinto del de ellos. Ella se ve investida con la responsabilidad de la economía de su hogar, lo cual la lleva a hacerse cargo de la producción de bienes de subsistencia y de la crianza de los hijos; por otra parte, su esposo estará obligado rápidamente a buscar trabajo fuera de la explotación de sus padres. Con el paso del tiempo esto resulta en una reducción del número de grupos domésticos que se dedican de manera exclusiva a la actividad agrícola. La actividad de produc- 
ción del henequén la realiza elijefe del grupo doméstico movilizando cuando mucho a uno de sus hijos solteros; los otros, así como los casados que todavía viven con él, se incorporan al mercado de trabajo no agrícola. Su mujer y sus nueras aseguran, cada una por su parte y para su propio núcleo doméstico, la producción de bienes alimenticios cerca de la casa.

El conjunto de la fuerza de trabajo del grupo doméstico es así movilizado de manera dispersa para asegurar la supervivencia del grupo, que existe cada vez menos como unidad de producción, perdurando más bien, en el mejor de los casos, como unidad de redistribución de la fuerza de trabajo de la cual dispone. Esta organización también es frágil porque el mercado de trabajo está constituido esencialmente por oportunidades puntuales creadas directa o indirectamente por las instituciones estatales. Estas posibilidades de trabajo, como las de jornalero o empleado en los sectores de la construcción o los servicios, permiten asegurar la supervivencia cotidiana del grupo doméstico, pero más a largo plazo sentencian, por su repetición y diversidad, las formas de organización colectiva del grupo, hasta entonces basadas en la unidad de producción agrícola. Los solteros y solterasijóvenes, al cubrir ellos mismos sus propios gastos, ya no intervienen ni física ni materialmente, o sólo de manera esporádica, en la economía del grupo doméstico. En cambio, se percibe una intensificación y diversificación de la actividad de las mujeres casadas. Su participación más fuerte en la producción agrícola, con el fin de asegurar la producción de subsistencia, las lleva a movilizar a sus hijos más que sus esposos. Esto permite entender en parte por qué en su discurso ellas aún se refieren, en proporción relativamente más alta que sus esposos, a los ideales de una descendencia numerosa. Esta actitud entra en contradicción con el hecho de que están conscientes de la necesidad de que sus hijos tengan una escolaridad continua y prolongada para que puedan encontrar trabajo en la ciudad (Gautier y Quesnel, 1993).

Entre producción agrícola e inserción urbana o la transformación del lugar del niño bajo la influencia de la escolarización

En África occidental el desarrollo de la escolarización modifica el antiguo equilibrio de las relaciones entre padres e hijos. Losijefes de familia que disponen de medios suficientes, particularmente los de zonas de agricultura comercial, escolarizan a sus descendientes al 
máximo, con el fin de que puedan insertarse en las esferas de actividad moderna. Conciben la escolarización como una inversión -desde luego incierta y en el largo plazo-, pero que puede parecer plenamente justificada en un periodo de ingresos elevados, de numerosos empleos urbanos y de cohesión familiar aún fuerte. Esta escolarización tiene un efecto importante en el medio rural, porque durante toda su escolarización, que en el África subsahariana puede ser bastante larga, el niño pasa de ser productor a ser consumidor. Más tarde su actividad tenderá a ejercerse de manera independiente a la de sus padres y, las más de las veces, en otros sectores productivos. La escolarización opera de cierta forma una reducción de la presión territorial, al menos provisionalmente. Así, la temporalidad y el contenido efectivo de los flujos de riqueza intergeneracionales tradicionales, hasta entonces en beneficio de los padres, se alteran: los padres ya no se benefician más que por las transferencias de bienes y servicios por parte de los hijos.

Este marcado desentendimiento de los hijos respecto a las unidades de explotación es decisiva, porque ejerce una desvinculación entre producción agrícola y reproducción demográfica, por la introducción de un nuevo término, la inserción urbana, en las dinámicas de las sociedades agrarias. Por esto el esquema de Chayanov, de ajuste de la producción al tamaño y a la estructura de la familia, no funciona en la medida en que sólo es pertinente si el conjunto de la fuerza de trabajo se invierte en la producción agrícola, o por lo menos si concurre en la supervivencia del grupo y mantenimiento de la explotación agrícola. Asimismo, el modelo de Boserup, de progreso técnico bajo el efecto de la presión demográfica, es inoperante en virtud del éxodo rural y de las migraciones hacia nuevos frentes pioneros, que representan un sustituto a la intensificación agrícola (Balac, 1994).

La escolarización transforma las aspiraciones de las generaciones ijóvenes y las empuja principalmente hacia los empleos urbanos, considerados modernos y rentables, para los cuales los prepara la escuela, suscitando así un éxodo rural pronunciado. Sin embargo, después de algunos años esta situación tiende a invertirse: por una parte, las salidas del mercado laboral se encuentran considerablemente reducidas y, por otra, la escolarización máxima de los niños se vuelve difícil de lograr, en virtud de la disminución en los ingresos de los campesinos. Además, a medida que la inversión educativa se vuelve más costosa y de rentabilidad más incierta, se desarrollan marcados fenómenos de desescolarización, así como de intentos de reinserción rural entre 
losijóvenes que se quedan sin empleo en la ciudad. No obstante, estas experiencias de regreso a la tierra resultan muy diffciles en virtud de los numerosos bloqueos de carácter territorial, financiero o técnico. Estos jóvenes, cuya movilización se facilita por el anterior establecimiento de redes bipolares ciudad-campo, constituyen la mayoría de las veces una mano de obra agrícola flotante de débil productividad, susceptible de emplearse temporalmente, aunque también de buscar una nueva inserción urbana. Son raros aquellos que, aptos para las innovaciones técnicas en virtud de su escolarización, se establecen como nuevos explotadores en el marco de un relevo campesino todavía hipotético (Affou Yapi, 1990; Faussey-Domalain y Vimard, 1991).

En Yucatán, en cambio, la escolarización de los hijos no entra en ningún momento en una estrategia de inversión a largo plazo, ni como medio de aligerar la presión territorial. Las familias de productores de henequén se mantienen al margen del sistema escolar, aunque se integran al sistema de salud moderna (Menéndez, 1981; Gautier y Quesnel, 1993). Hasta finales de los años sesenta los jóvenes, escolarizados o no, se desplazaban hacia el medio urbano más cercano y hacia otros estados vecinos, donde buscaban emplearse como mano de obra, sobre todo en la construcción. A pesar de la débil dimensión del espacio migratorio, las bajas oportunidades de empleo que encierra y el mantenimiento de los niños fuera del sistema escolar, éstos son otros tantos elementos que pueden explicar por qué mientras se deterioran las condiciones de la producción del henequén, se presencian paradójicamente la conservación de las unidades domésticas en la esfera de la producción agrícola y la retención de una mayoría de la población en las zonas rurales. Este doble movimiento, llamado recampenización, ha alimentado durante un tiempo la ilusión de que un campesinado pequeño quería y podía vivir de la producción de sus parcelas según el modelo de Chayanov. Ahora bien, si es cierto, como se verá más adelante, que la distribución de las actividades está ligada a la etapa del ciclo demográfico del grupo doméstico en un momento dado, es necesario notar que es la viabilidad de las explotaciones agrícolas lo que está estructuralmente cuestionado. Es necesario además subrayar que es en virtud de la creciente participación de las mujeres en la actividad agrícola, en principio para asegurar la subsistencia del grupo -y en otros sectores, como el artesanal, para asegurar la crianza de sus hijos-, que éstas han podido mantenerse en su grupo de origen ejerciendo una actividad fuera de él. Así, las generaciones más jóvenes ya no participan en la producción agrícola. 
En definitiva, las posiciones asignadas a los niños en la familia, y más globalmente en la sociedad, están definidas cada vez menos por su participación en la producción agrícola. Dependen sobre todo de elementos exteriores a dicha producción: la escolarización y los sectores modernos de actividades, y esto con una intensidad creciente, según la antigūedad de la inserción de la población en la economía agrícola mercantil. Se presencia así una diversificación de la posición y del papel de los niños en la reproducción social, correspondiente, por otra parte, a la diversidad de la integración de los niños en su propio hogar de residencia.

Crecimiento demográfico y restructuraciones familiares o los efectos de la transición demográfica

\section{La disminución de la mortalidad como acelerador de las transformaciones familiares y agricolas}

En las regiones rurales africanas la disminución de la mortalidad se generalizó a partir de los años sesenta. ${ }^{15}$ Esta disminución modifica la estructura de las diferentes instancias sociales, particularmente la de las unidades familiares, donde la coexistencia de varias generaciones y de más parientes colaterales durante periodos más largos perturba los ciclos de transmisión de poderes y bienes, mientras que el incremento de los descendientes aumenta los gastos de crianza de los niños. De pronto se acelera la segmentación de las grandes unidades familiares, implicando una redefinición del uso de tierras y de la división del trabajo entre hombres y mujeres y entre las generaciones comprendidas en las nuevas unidades. Entonces, cuando antes los hombres solteros jóvenes de las grandes unidades familiares se ausentaban por rotación durante periodos relativamente cortos (de las regiones de cultivos comerciales hacia los empleos urbanos o de las regiones productoras de cultivos alimenticios de subsistencia hacia las agriculturas comerciales o hacia las ciudades), hoy en día una parte

${ }^{15}$ La disminución de la mortalidad en el África subsahariana es más importante en las regiones de economía de plantación, en cuyas zonas más desarrolladas el nivel de mortalidad de niños entre 0 y 5 años de edad es solamente de 100 a 120 por cada mil desde principios de los años 70 . Én cambio, en las regiones de sabana desunadas a cultivos alimenticios este nivel, todavía en los años 80 , alcanzó 230 por cada mil. 
creciente de las nuevas generaciones emigra (Boutillier et al, 1977; Quesnel y Vimard, 1987). Esta emigración diferencial acentúa, particularmente en las zonas de cultivos de subsistencia y en las regiones de plantación antigua, cambios agrícolas en el sentido de una degradación de los sistemas de explotación: feminización de la producción de subsistencia, aumento de los costos del trabajo de las mujeres y envejecimiento de losijefes de la explotación, última característica especialmente desfavorable para las innovaciones agrícolas

Asimismo, el crecimiento numérico de la población, que exige un aumento de la producción agrícola, se traduce en una extensión de las superficies cultivadas, conduciendo en el largo plazo al deterioro de las condiciones de funcionamiento de estos sistemas de explotación: saturación territorial, reducción de los periodos de barbecho, disminución del rendimiento... Respecto al crecimiento del número de niños, provoca conflictos en cuanto a la distribución de los gastos que vuelven más inestables las unidades familiares, particularmente en tanto que la crisis económica se exacerba, contribuyendo a acelerar un cuestionamiento de los ideales de la fecundidad.

La evalución de la fecundidad como reveladora de las incertidumbres sociales y fuente de innovaciones y reajustes agricolas

El desarrollo de la escolarización en las sociedades rurales coloca al nino en una nueva trayectoria, y esto induce profundos cambios en las actitudes hacia la fecundidad. El marco ideológico tradicional de la fecundidad, que favorece una descendencia numerosa, aduciendo distintasijustificaciones (necesidad de mano de obra, sostén de los ancianos, permanencia de los grupos sociales...), puede verse ahora cuestionado en sus concretizaciones y aun más en sus referencias, y tanto más intensamente cuanto que la desherencia de los poderes del linaje se verifica por su pérdida de control de la reproducción física. Este cuestionamiento no es, sin embargo, general, y pueden observarse fenómenos inversos, particularmente desde el punto de vista de las mujeres. Así, entre los moba-gurma del norte de Togo, la autonomía de la mujer y la participación creciente que debe asumir en la producción agrícola la conducen a querer aumentar su descendencia, para poder beneficiarse de una mano de obra más numerosa. En este caso se presencia lo que podría considerarse como una perversión del esquema de Boserup, en la medida en que estas mujeres dedicadas a la producción agrícola de subsistencia producen innovaciones agrícolas que tie- 
nen las más de las veces el objetivo de reducir las restricciones de la mano de obra y que conducen a reforzar los factores de un crecimiento demográfico más fuerte. Pero es en el dominio social donde se ve la aparición de prácticas familiares innovadoras, como el recibimiento de niños por parte de mujeres jóvenes que se encuentran todavía en una fase de constitución de su descendencia (Rey, 1989).

El papel del niño y su lugar en las estrategias sociales y agrícolas no están fijadas de manera definitiva y son susceptibles de ajustes y retrocesos, según la coyuntura económica, las posibilidades de recurrir a una mano de obra externa a la familia nuclear y la idea que se hacen los padres de la validez de la escolarización como inversión. También la evolución de la fecundidad en el África rural es afectada por diversas tendencias, en relación con el costo de los hijos y los beneficios, inmediatos o aplazados, esperados por la familia, y en función de las estrategias sociales de las diferentes poblaciones.

Por otra parte, estas relaciones no son unívocas, pues los ideales de una elevada fecundidad pueden corresponder a estrategias dirigidas ya sea hacia una simple reproducción de la sociedad agrícola (es el caso tanto de los aparceros kabye que emigran a la meseta de Daye en Togo como de los agricultores de Sassandra en el oeste de Costa de Marfil) o, por el contrario, hacia una extraversión a las esferas urbanas y estatales (es así entre los akye del sudeste de Costa de Marfil durante periodos de crecimiento económico); por consiguiente, pueden corresponder a una orientación de los niños hacia el trabajo agrícola o hacia la escolarización. ${ }^{16}$

Pero la crisis económica, que tiende a aumentar el costo de los niños, provoca en ciertas poblaciones una presión para disminuir la fecundidad, como se ha podido constatar entre los ewe autóctonos de Daye y como se comienza a percibir entre los akye de Costa de Marfil. Esta voluntad de reducción de la fecundidad también es claramente perceptible entre los obreros agrícolas que venden su fuerza de trabajo en los grandes complejos agroindustriales del suroeste de Costa de Marfil (Guillaume y Vimard, 1994). A pesar de esto, la disminución de la descendencia no es siempre efectiva e inmediata, en virtud de

${ }^{16}$ De hecho, una población puede conservar actitudes favorables hacia una elevada fecundidad mientras continúa cubriendo los costos de los niños que desea dirigir hacia los empleos urbanos para un beneficio a largo plazo. En este caso el jefe de la familia busca una fuerte descendencia en la medida en que "entre más niños haya, más posibilidades hay de que alguno tenga éxito". 
la débil oferta de medios anticonceptivos modernos en la mayor parte de los países africanos y de que se sigan menos las prácticas tradicionales de espaciar los nacimientos. Además, las dificultades de recurrir a un salariado agrícola, que por lo regular se vuelve demasiado oneroso por la baja en los ingresos de los cultivos comerciales, imponen un repliegue sobre una mano de obra familiar (Léonard, 1993); repliegue que conduciría a ciertosijefes de explotación a querer continuar disponiendo de una elevada descendencia.

En definitiva, se perciben actualmente evoluciones contrastantes de la fecundidad en las diferentes regiones rurales. Si bien la introducción de las poblaciones del África subsahariana a la esfera de la economía de mercado crea una disociación en tre fuerza de trabajo y reproducción demográfica, que transforma el papel de los niños, los costos de su educación y los beneficios que se esperan de ella, el ideal de una elevada fecundidad aún no está profundamente alterado (salvo en ciertos grupos urbanizados e instruidos), a pesar de ciertas transformaciones de sus justificantes y de las prácticas que les corresponden. No obstante, la amplificación de la crisis económica y social replantea la cuestión de la descendencia en nuevos términos, suscitando los fermentos de un cuestionamiento del ideal de fecundidad elevada, incluso si la falta de contracepción moderna contraría su traducción en índices de una nueva problemática demográfica (Vimard et al., 1994).

Fue así en Yucatán. La crisis económica alteró fuertemente el marco de referencia de los ideales y conductas en materia de fecundidad. Los ideales transmitidos por las mujeres se inscriben en lo sucesivo en un marco económico en el corto plazo. Sin embargo, la formalización de una demanda de regulación de la fecundidad no hubiera podido establecerse tan rápido sin la presión ideológica y material de los agentes de las instituciones de salud. Para legitimar la planificación familiar estos últimos retoman el discurso de las familias sobre el deterioro de su economía doméstica; difunden las normas biomédicas de la procreación y ofrecen medios de contracepción al momento del control prenatal (Gautier y Quesnel, 1993).

Transformación de los modelosifamiliares en relación con el control de los medios de producción agricola

Con el rejuvenecimiento de las estructuras demográficas y el crecimiento de la población en el medio rural africano (más de $2 \%$ anual 
durante la década de los ochenta), resultado de la disminución de la mortalidad y del mantenimiento de una elevada fecundidad, ${ }^{17}$ lo que se ve modificado son las condiciones demográficas de reproducción de las relaciones sociales tanto dentro de las familias africanas como entre ellas.

La fragmentación del linaje en unidades autónomas en cuanto a su producción agrícola y reproducción física está acompañada por una nuclearización relativa de los grupos domésticos. No obstante, a pesar de esta primacía del componente nuclear (el hombre, sus esposas, sus hijos), el hogar nuclear, correspondiente a la reducción del grupo residencial al núcleo único de reproducción biológica, no es más que uno de los modelos residenciales adoptados por las diferentes poblaciones rurales. De hecho, otros fenómenos determinan una pluralidad morfológica de los hogares revelando nuevas líneas de fuerza de los modelos familiares: la integración de los nietos y el recibimiento de parientes colaterales, ${ }^{18}$ que marca la dimensión extranuclear del grupo doméstico, o la inestabilidad matrimonial, que conduce a una fragmentación de la célula conyugal, originando familias monoparentales y una intensa redistribución de los niños entre los hogares. Estos fenómenos, frecuentes en numerosas poblaciones, revelan la adaptación de las solidaridades anteriores para mitigar las diversas desestabilizaciones de la organización matrimonial y familiar ${ }^{19}$ (Quesnel y Vimard, 1989).

La articulación de estos fenómenos diseña una multiplicidad de configuraciones familiares de las cuales se desprenden dos modelos dominantes ligados al modo de control territorial y al grado de adecuación entre unidad de explotación y grupo doméstico. El primer modelo consiste en un grupo doméstico de forma diversificada y de estructura inestable en virtud de la movilidad, la extraversión hacia el

${ }^{17}$ La esperanza de vida pasó en África de 38 años en 1950-1955 a 53 años en 1990, expresando asi el retroceso de la mortalidad durante tres décadas, mientras que la descendencia de las mujeres en esta fecha sigue comprendida entre 6 y 7 hijos en la mayor parte de los países.

${ }^{18}$ En ciertas poblaciones los nietos y parientes colaterales pueden representar hasta $30 \%$ de los individuos de un hogar.

${ }^{19}$ Las redes de asistencia que se establecen así tienen por otra parte el efecto de conectar el mundo rural con el urbano y de permitir que las familias realicen entre sí distintos intercambios (de dinero, alimentos...), sin olvidar a los individuos mismos que se desplazan de un polo a otro según ciertas redes, particularmente familiares; redes que permiten particularmente la circulación de una mano de obra agrícola temporal (Lesourd, 1987). 
mundo urbano y estatal y la crisis agrícola y económica endógena y exógena. Esta estructura caracteriza a las comunidades antiguamente insertadas en la economía de plantación, como las poblaciones autóctonas de Daye y los akye del suroeste de Costa de Marfil, donde la desagregación relativa del tejido demográfico se acompaña con una dispersión del patrimonio territorial y una relativa inadecuación entre el grupo de explotación y el grupo doméstico o residente de los parientes desvinculados de la producción agrícola.

El segundo modelo se define por el mantenimiento de un grupo doméstico estable, compuesto por una sola o varias familias nucleares (en cuyo caso los jefes de familia son hermanos o padre e hijo) con otros parientes (colaterales, ascendientes...). Este tipo es en particular el de los grupos kabye que emigran a Daye y el de los agricultores independientes de Sassandra, entre quienes el patrimonio territorial y la mano de obra familiar residente en el grupo doméstico permanecen controlados por los:jefes de grupo (Vimard, 1993); un control sobre la fuerza de trabajo familiar que tiende, por otra parte, a reforzarse en los periodos de crisis.

La organización de los grupos domésticos en la zona henequenera de Yucatán es de cierta manera la consecución del primer modelo, incluso si las configuraciones familiares parecen deducirse del segundo. De hecho, si bien la nuclearización de los grupos domésticos es relativamente débil ${ }^{20}$ y parece confirmar el modelo familiar patrilineal y de residencia plurigeneracional, hay que recordar que hay una multiplicación de componentes nucleares debido a la disminución de la mortalidad. Estos componentes, al tener una economía doméstica y una actividad independientes de las de su padre y sus colaterales, no pueden, por las mismas razones económicas que les hicieron separarse de la producción agrícola, constituir una unidad residencial independiente. Los hombres casados dejan el grupo doméstico de origen en cuanto pueden, como lo indica la fuerte nuclearización de los grupos domésticos encabezados por hombres de entre 25 y 40 años de edad. ${ }^{21}$ En cambio, la generación de más de 40 años de edad, que vivió la transición demográfica, presenta arreglos muy complejos que

\footnotetext{
${ }^{20}$ Sólo $55 \%$ de los grupos domésticos son nucleares, y sólo $28 \%$ lo son si se toma en cuenta el hecho de que el padre sobreviva (en otras palabras, el otro $27 \%$ quizás sean nucleares debido a la muerte del padre).

${ }^{21}$ Cuando el padre vive, más de $62 \%$ de los grupos domésticos cuyo jefe tiene entre 25 y 29 años de edad son nucleares, y cerca de la mitad cuando tiene entre 30 y 39 años.
} 
subrayan el choque de las relaciones económicas intergeneracionales: los hombres de estas generaciones pueden integrar en su grupo doméstico a sus padres o a su madre viuda, al componente nuclear de uno de sus hijos casados, a parientes colaterales solteros, etc. Se comprende por lo tanto que el ciclo demográfico de un grupo tal esté vinculado a las coyunturas económicas y sociales, y no a un modelo familiar organizado en torno a un objetivo de producción.

Las mutaciones familiares como constreñimiento de las innovaciones agrícolas

La nueva dependencia de las unidades de producción con respecto al ciclo de vida familiariy al régimen demográfico

Si bien en las poblaciones autóctonas los productores agrícolas se desprenden de la dependencia familiar y de linaje en cuanto a la producción, se enfrentan rápidamente con la carga que representa la crianza de sus hijos y con la pérdida de mano de obra debida a la escolarización de una parte de ellos. Son obligados entonces a movilizar a sus esposas y a una población alóctona (en las zonas de cultivos comerciales) para obtener la fuerza de trabajo que necesitan. En el largo plazo esta doble exigencia ha sido portadora de tensiones y luego de bloqueos socioeconómicos que, conjugados con el rápido crecimiento demográfico, han conducido a las poblaciones agrícolas a perpetuar el recurso a la solución migratoria y a una creciente extraversión de sus actividades hacia la ciudad o hacia las nuevas zonas de cultivo.

En los grupos alóctonos la estructura de los poderes y el ciclo demográfico de las familias están condicionados por las transformaciones del proceso de reproducción social de las poblaciones autóctonas: es la posibilidad que tienen de hacer trabajar a las mujeres y niños (poco escolarizados) en sus explotaciones, lo cual ofrece a los responsables de los grupos domésticos alóctonos la oportunidad de instalarse de manera estable en las regiones de plantación. Esta situación sigue siendo precaria, pues todavía depende en gran medida de la no escolarización de los hijos, de la disponibilidad de tierras vírgenes y de la necesidad de los jefes de familias autóctonas de recurrir a una fuerza de trabajo suplementaria. Así, cada vez que no se reúnen estas condiciones, se amenaza la instalación estable de los alóctonos, 
activando con ello nuevos desplazamientos hacia los frentes pioneros o los medios urbanos.

Sea como fuere, existe, por un lado, una dependencia recíproca entre los ciclos de vida demográficos de las familias autóctonas y alóctonas (es en particular la retención de los jóvenes alóctonos lo que permite la fuerte movilidad y escolarización frecuente de los jóvenes autóctonos), que no hace sino acentuar la diferenciación de los ciclos de vida familiares entre estas dos poblaciones. Se opera, por el otro lado, una segunda dependencia de los sistemas de explotación respecto a los ciclos demográficos y las estrategias sociales de las diferentes poblaciones presentes.

Así, la inserción de las comunidades rurales en el sistema de economía mercantil se traduce en la emergencia de nuevas relaciones sociales de producción y de reproducción demográfica. Más allá de la independencia del grupo doméstico y de su nuclearización relativa, se observa la emergencia de una jerarquía inestable y compleja de las familias y una creciente autonomía de los individuos en la producción y reproducción, dos fenómenos que son portadores a largo plazo de reorientaciones inéditas de las prácticas de los actores sociales, tanto en la esfera familiar como en el sistema de producción agrícola, de las cuales constituyen una dinámica propia.

Las innovaciones agrícolas bajo el constreñimiento de las continuas recomposiciones familiares

Con un crecimiento demográfico que haya permanecido débil durante un largo periodo, el ciclo de vida demográfico podría institucionalizarse en generaciones y grupos de edad; es a la vez constitutivo y específico de la sociedad en cuestión. La presión demográfica, mientras no amenace a las instituciones, podría dar lugar a innovaciones tanto técnicas, según el esquema de Boserup, como sociales. Pero ante un crecimiento demográfico rápido, que acompaña a la inserción más marcada $d$ e las sociedades agrarias en la economía de mercado, las instituciones que rigen la producción han visto su estructura y su ciclo demográfico redefinirse constantemente siguiendo las oportunidades exteriores a la producción agrícola. Los ajustes se definen entonces en el campo de lo social y a corto plazo. Así, la extensión de las superficies cultivadas (y el aumento de la producción agrícola en una primera etapa de la transición demográfica) responde menos a una fragmentación 
de las explotaciones bajo la presión demográfica que a una recomposición continua de las unidades domésticas y de las unidades de explotación. Todas estas transformaciones se traducen en la estructura de explotación agrícola por un aumento de la proporción de población de más de 50 y menos de 15 años de edad y por un desequilibrio creciente en la proporción entre sexos, en beneficio de las mujeres.

Las mujeres adquieren así un papel preponderante en la producción agrícola y particularmente en la producción alimenticia, que, por otra parte, está en auge por la demanda citadina. Este papel creciente se prolonga en la transformación y comercialización de los productos alimenticios, lo cual aumenta su participación en la obtención de los ingresos del hogar (Adjamagbo, 1995; Chaléard, 1996). Ellas son capaces de innovaciones de orden técnico y muy receptivas a las innovaciones procedentes del exterior cuando se inscriben en esta misma problemática de maximización de los ingresos, obtención de seguridad alimenticia y equilibrio de las cargas de trabajo en el tiempo. No obstante, al incorporarse de manera creciente a la producción agrícola y al buscar siempre minimizar sus sobrecargas de trabajo, las mujeres se ven obligadas a adoptar prácticas culturales que afectan directamente a la productividad de la tierra (aprovechamiento de tierras marginales, reducción del tiempo de barbecho...) y que se revelan como perjudiciales al ambiente. Deben recurrir igualmente, más que en el pasado, al trabajo de sus hijos, lo cual refuerza sus prácticas reproductivas en favor de una descendencia numerosa. Se orientan así hacia situaciones límite portadoras de rupturas, una de las cuales puede ser la migración hacia la ciudad, que afecta fuertemente la producción alimenticia.

También losijóvenes son llevados a adoptar estrategias más individuales ante las dificultades económicas que atenazan a las explotaciones agrícolas, así como ante el retraso en la transmisión de los bienes territoriales y su parcelación entre múltiples colaterales. Éstas se traducen de diferentes maneras: producción agrícola en superficies prestadas por los padres, peonaje retribuido en grupos de ayuda mutua reconstituidos, pluriactividad o circulación creciente entre el campo y la ciudad, donde algunos se instalan, inscribiéndose entonces fuera de la esfera de la producción agrícola (Quesnel y Vimard, 1988; Faussey-Domalain y Vimard, 1991).

Esta elasticidad de arreglos y comportamientos aparece como un freno a lo que debería ser un proceso de innovación lógica y lineal 
bajo la presión demográfica. Ésta es, por el contrario, fuente de reorientaciones inéditas del sistema de explotación. Las mujeres y los jjóvenes desarrollan una gran capacidad de innovaciones sociales que aspiran sobre todo a superar los bloqueos territoriales y las restricciones de la mano de obra. Parece que es en el seno del sistema de cultivos donde repercuten las perturbaciones y la transformación del sistema de explotación familiar, dominio en el que se ha notado una gran capacidad de adaptación de las agriculturas africanas (Couty, 1991). Es importante subrayar que, de manera general y como se ha visto a lo largo del texto, las innovaciones son las más de las veces y en principio generadas al interior; no obstante, en estas condiciones no puede encontrarse tiempo y espacio para innovaciones cuyo resultado sería preservar la explotación y mejorar la productividad del trabajo y de las tierras.

También es importante que los organismos de desarrollo que quieran introducir nuevas tecnologías y variedades de cultivos en la agricultura tomen más en cuenta el contexto institucional de la producción agrícola y, sobre todo, la dinámica de recomposición de las unidades domésticas y de explotación agrícola, así como la participación de los diferentes individuos de la familia en esta producción.

\section{Bibliografia}

Adjamagbo, A. (1995), "De l'expression des solidarités familiales dans les societés d'économie de plantation", Seminario internacional Ménage et famille en Afrique: bilan, enjeux et perspectives de recherche, CEPED-ENSEAINS-ORSTOM-URD, Lomé, 4-9 de diciembre de 1995 (mimeo.).

Affou, Y.S. (1990), La relève paysanne en Côte d'lvoire, Paris, Karthala/orstom.

Augé, M. (1971), "Traite précoloniale, politique matrimoniale et stratégie sociale dans les sociétés lagunaires de basse Côte d'Ivoire", Cahiers de l'onstom, vol. 8, núm. 2 (Serie Ciencias Humanas), pp. 143-152.

Balac, R. (1994), "L'acheminement du système d'économie de plantation vers un blocage structurel. Eléments d'analyse d'une crise", Coloquio Crises, djustements et recompositions en Côte d'lvoire: la remise en cause d'un modèle?, Abidjan, gids-orstom, 28 de noviembre-2 de diciembre, (mimeo.).

Bernard, C. y S. Gruzinski (1986), "Les enfants de l'Apocalipse: la famille en Méso-Amérique et dans les Andes”, en Burguière, A., C. Klapisch-Zuber, M. Segalen y F. Zonabend (eds.), Histoire de la famille, tomo 2, Paris, Armand Colin, pp. 157-209. 
Boserup, E. (1970), Evolution agraire et pression démographique, París, Flammarion [el original en inglés es de 1965].

(1983), La femme face au développement économique, París, PUF.

(1991), "Causes and Effects of Disequilibria in Food Production", en Gendreau, F. C. Meillassoux, B. Schlemmer y M. Verlet (eds.), Les spectres de Malthus, París, EDI-ORSTOM-CEPED, pp. 33-40.

Boutillier, J.L., A. Quesnel y J. Vaugelade (1977), “Systèmes socio-économiques mossi et migrations", Cahiers de l'orstom, vol. 14, núm. 4 (Serie Ciencias Humanas), pp. 361-381.

Capron, J. y J.-M. Kohler (1975), "Migrations de travail et pratique matrimoniale", Ouagadougou, orstom (mimeo.).

Chaléard, J.-L. (1996), Temps des villes. Temps des vivres. L'essor du vivrier marchand en Côte d'Ivoire, Paris, Karthala.

Chayanov, A. (1990), L'organisation de l'économie paysanne, Paris, Librairie du Regard [el original en inglés es de 1966].

Couty, P. (1991), "L'agriculture en réserve. Réflexions sur l'innovation et l'intensification agricoles en Afrique tropicale", Cahiers d'études africaines, vol. 31, núm. 121-122, pp. 65-81.

Faussey-Domalain, C. y P. Vimard (1991), “Agriculture de rente et démographie", Revue Tiers-Monde, vol. 32, núm. 125, pp. 93-114.

Fauve-Chamoux, A. (1987), Evolution agraire et croissance démographique, Lieja, Ordina Editions.

Fiéloux, M. (1985), "Développement, émigration masculine et travail féminin, le cas des femmes toucouleur de la région du Demga (Moyenne vallée du Sénegal)", en Femmes et politiques alimentaires, Paris оrsтом (Col. Coloquios y Seminarios), pp. 328-345.

Gautier, A y A. Quesnel (1993), Politique de population, médiateurs institutionnels er rá gulation de la fecondité au Yucatán (Mextique), París, Orstom (Col. Estudios y Tesis).

Gendreau, F., C. Meillasoux, B. Schlemmer y M. Verlet (eds.) (1991), Les spectres de Malthus, París, ORSTOM-EDI-CEPED.

Guillaume, A. y P. Vimard (1994), "Fecondité, crise économique et différentiations sociales à Sassandra (sud-ouest de la Côte d'Ivoire)", en N. Koffi, A. Guillaume, P. Vimard y B. Zanou (eds.), Maîtrise de la croissance démographique et développement en Afrique. Séminaire ENSEA-ORSTOM, Paris, ORSTOM (Col. Coloquios y Seminarios), pp. 143-168.

Le Cour Grandmaison, C. (1979), "Contrat économique entre époux dans l'Ouest africain", L'Homme, vol. 19, núms. 3-4, pp. 159-170.

Léonard, E. (1993), "Différentiation et reproduction des exploitations agricoles dans le Sud-Ouest ivoirien: une typologie des exploitations", Abid:jan, ORSTOM (mimeo.).

Lerner, S. y A. Quesnel (1985), "La estructura familiar como expresión de condiciones de reproducción social y demográfica. El caso de la zona henequenera en Yucatán", Reproducción de la población y desarrollo, vol. 5, Buenos Aires, Clacso, pp. 157-212. 
(1989), "El espacio familiar en la reproducción social: grupos domésticos residenciales y de interacción", en Olliveira et al. (eds.), Grupos domésticos y reproducción cotidiana, México, Porrúa/El Colegio de México, pp. 39-79.

Lesourd, M. (1987), "La forêt, la machette et le billet de banque", Cahiers de l'orstom, vol. 24, núm. 1 (Serie Ciencias Humanas), pp. 73-97.

Marchai, J.Y. (1987), "En Afrique des savanes, le fractionnement des unités d'exploitation rurales ou le chacun pour le soi", Cahiers de l'onstom, vol. 23, núm. 3-4 (Serie Ciencias Humanas), pp. 445-454.

Menéndez, E.L. (1981), Poder, estratificación y salud. Análisis de las condiciones sociales y económicas de la enfermedad en Yucatán, México, Ediciones de la Casa Chata.

Pantelides, E. (1984), "Familia y fecundidad: balance y perspectivas en el caso latinoamericano", en Memorias del Congreso latinoamericano de población y desarrollo, vol. 2, México, uNAM/El Colegio de México/PISPAL, pp. 675-693.

Paulme, D. (1952), "La femme africaine au travail", en Le travail en Afrique noir, Paris, Seuil (Presence Africaine), p. 121.

Pilon, M. (1989), Enquête socio-démographique chez les Moba Gurma du Nord-Togo, vol. 2: Caractéristiques et évolution des ménages, Lomé, оRsтом.

(1991), "Genèse du déséquilibre entre population et ressources en pays moba gurma (Nord-Togo)", en Gendreau, F., C. Meillassoux, B. Schlemmer y M. Verlet (eds.), Les spectres de Malthus, Paris, edtorstom-CEPED, pp. 117-136.

Quesnel, A. y P. Vimard (1987), "Système de production et dynamique de populations en économie de plantation", Cahiers de l'orstom, vol. 23, núms. 3-4 (Serie Ciencias Humanas) pp. 483-503.

(1988), Dynamique de population en économie de plantation. Le plateau de Dayes au sud-ouest du Togo, París, orstom (Col. Estudios y Tesis).

- (1989), "Famille plurielle en milieu rural africain", Cahiers de $l$ orstom vol. 25, núm. 3 (Serie Ciencias Humanas), pp. 339-355.

Rey, S. (1989), "'Entre avec la fraîcheur dans la maison' Schémas de reproduction et de santé, évolution du statut des femmes et transformations des milieux en pays moba-gurma (Nord-Togo)", tesis de doctorado, Marsella, Laboratorio de Población y Ambiente, Universidad de Provence.

Vimard, P. (1993), "Modernité et pluralités familiales en Afrique de l'Ouest", Revue Tiers-Monde, vol. 34, núm. 133, pp. 89-115.

Vimard, P., A. Guillaume y A. Quesnel (1994), "Singular Fertility Patterns in Rural Africa. Socio-Economie Differentiations and Transformation of Fertility Models in West Africa", en Locoh, T. y V. Hertrich (eds.), The Onset of Fertility Transition in sub-saharan Africa, Lieja, Ordina Editions, pp. 193-220. 
